\title{
Konzepte und Effekte familienbildender Angebote für Migranten zur Unterstützung frühkindlicher Förderung
}

\section{Concepts and effects of parent education programs for supporting early childhood education in immigrant families}

\begin{abstract}
Zusammenfassung:
Im Kontext des Diskurses über den Abbau der Bildungsbenachteiligung von Kindern und Jugendlichen aus Familien mit Migrationshintergrund wird der Blick verstärkt auch auf die Möglichkeiten der Familienbildung gerichtet. Hier wurden in den letzten Jahren zahlreiche Konzepte entwickelt bzw. aus dem Ausland adaptiert. Im vorliegenden Aufsatz werden solche Programme anhand eines mehrdimensionalen Kategorienschemas systematisiert. Als Unterscheidungskriterien wurden die Anzahl der Programmmodule, die Adressaten sowie die Konzeptstruktur herangezogen. Ergänzende Analysen verdeutlichen, dass sich die meisten Programme sehr um Niedrigschwelligkeit bemühen und auch kurzfristig zu positiven Effekten führen. Inwieweit durch Familienbildung tatsächlich langfristige Effekte auf den Bildungserfolg der Kinder erzielt werden können, lässt sich auf Grundlage vorliegender empirischer Untersuchungen jedoch nicht beurteilen, da diese in der Regel weder ein Kontrollgruppen- noch ein Längsschnittdesign aufweisen.
\end{abstract}

Schlagwörter: Frühkindliche Förderung, Familienbildung, Migranten, Effekte

\begin{abstract}
:
As a consequence of the discourse on the ways and means of reducing educational disadvantages of immigrant children and youth in Germany, family education and parent education programs have increasingly drawn attention as possible solutions to these problems. During recent years, in this field numerous programs have been either developed domestically or adapted from other programs originating outside of Germany. In this article, some of these programs are systematically classified by employing a multi-dimensional scheme. As criteria of distinction, the number of program modules, target groups and program structures are taken into account. Further analyses indicate that most programs care a lot about a low-threshold access and that they do produce positive short-term effects. Based on existent empirical studies, it is, however, not feasible to assess long-term effects on the children's scholastic advancement, since in general these studies neither involved a control group nor did they include longitudinal research.
\end{abstract}

Key words: early childhood education, family education programs, immigrants, effectiveness 


\section{Einleitung}

Die Reduzierung der Bildungsbenachteiligung von Kindern und Jugendlichen aus Migrantenfamilien ist ein zentrales integrationspolitisches Anliegen der Aufnahmegesellschaft (Bundesregierung 2007). Mögliche Ansatzpunkte zur Schaffung besserer Bildungschancen für diese Gruppe sind das vorschulische Bildungs- und Betreuungssystem, das Schulsystem sowie die Familie. Vor allem die Familie als primärer Bildungsort ist bei der Diskussion um geeignete Strategien zunehmend in den Fokus gerückt. In diesem Kontext hat auch die Familienbildung an Bedeutung gewonnen (Bundesregierung 2007; Wissenschaftlicher Beirat 2002: 9). Familienbildende Angebote sollen dazu beitragen, dass Kinder in der Familie schon früh in schulrelevanten Kompetenzbereichen gefördert werden. Diese Angebote sollen insbesondere diejenigen Familien mit Migrationshintergrund erreichen, bei denen Faktoren, wie z.B. die Zugehörigkeit zu einer niedrigen sozialen Schicht, eine geringe Ressourcenausstattung oder ,ein nicht dem Mainstream entsprechender kultureller Hintergrund" (Leseman 2008: 128) dazu führen, dass die Kinder auf den Eintritt in das formale Bildungssystem weniger gut vorbereitet sind. Wenngleich diese Konstellation bei Familien mit Migrationshintergrund häufiger zu beobachten ist als bei Familien ohne Migrationshintergrund, sollte die Heterogenität dieser Bevölkerungsgruppe unbedingt im Blick behalten werden.

Ziel des vorliegenden Beitrags ist es, familienbildende Angebote für Migranten zur frühkindlichen Förderung auf der Basis eines mehrdimensionalen Kriteriensystems zu kategorisieren sowie anhand vorliegender empirischer Belege hinsichtlich ihrer Effekte zu untersuchen. Da Evaluationsergebnisse nicht ohne Weiteres auf andere Länder bzw. andere kulturelle Kontexte übertragen werden können (Lösel/Runkel 2012: 276), liegt der Fokus dabei auf Studienergebnissen aus dem deutschen Raum. Im ersten Kapitel wird zunächst die Notwendigkeit einer möglichst früh einsetzenden Förderung von Kindern aus benachteiligten Familien mit Migrationshintergrund begründet. Das zweite Kapitel richtet seinen Blick auf die institutionelle Familienbildung als präventives Angebot der Mehrheitsgesellschaft und begründet die Konzeption spezieller Angebote für die Zielgruppe der Migranten. Das dritte Kapitel ist der Kategorisierung und Analyse von Angebotskonzepten für die Zielgruppe der Migranten sowie der Frage nach den Effekten familienbezogener Interventionen zur frühkindlichen Förderung gewidmet. Abschließend werden im vierten Kapitel die wesentlichen Ergebnisse der Arbeit diskutiert.

\section{Die Bedeutung der Familie für den schulischen Bildungserfolg}

Folgt man Boudon (1974), so sind herkunftsbedingte Bildungsungleichheiten auf zwei Effekte zurückzuführen: Den primären und den sekundären Herkunftseffekt.

Der primäre Herkunftseffekt bezieht sich auf die Sozialisationsbedingungen in der Familie, welche in Abhängigkeit von der sozialen Positionierung der Familie sowie deren Ressourcenausstattung variieren. So verfügen Eltern mit einem höheren Sozialstatus und Bildungsniveau über bessere Möglichkeiten, ihren Kindern schon früh entwicklungsförderliche Anregungsbedingungen und Lernerfahrungen sowie schulrelevante Wissensinhalte zu vermitteln, als Eltern mit einem niedrigeren Sozialstatus und Bildungsniveau. In 
Bezug auf Familien mit Migrationshintergrund ist überdies zu fragen, inwieweit die Kinder dort unter spezifischen Erziehungs- und Sozialisationsbedingungen aufwachsen, welche mit den Migrationserfahrungen bzw. dem kulturellen Hintergrund der Eltern zusammenhängen. Empirische Analysen von Betz (2006) auf Basis der Daten des DJI-Kinderpanels zeigen unter Kontrolle der Milieuzugehörigkeit signifikante Unterschiede zwischen den kindlichen Lebens- und Erfahrungswelten von türkisch geprägten, russisch geprägten und Familien ohne Migrationshintergrund. Neben dem kulturellen Hintergrund lassen sich mindestens drei weitere Merkmale ausmachen, „die eher auf Familien mit Migrationshintergrund zutreffen als auf, einheimische“ " (Krüger-Potratz 2004: 8, Hervorhebungen im Original): Aufenthalts- oder arbeitsrechtliche Vorschriften, Mehrsprachigkeit $^{1}$ sowie transnationale Netzwerk- und Fürsorgebeziehungen zwischen in unterschiedlichen Ländern lebenden Familienmitgliedern. Es ist zu vermuten, dass sich diese Unterschiede in den Lebenswelten auch in den kindlichen Sozialisationsbedingungen widerspiegeln. Empirische Untersuchungen hierzu gibt es jedoch kaum. „Die häuslichen Erfahrungswelten von Kindern aus zugewanderten Familien sind nach wie vor ein weißer Fleck in der Forschungslandschaft" (Leyendecker 2008: 93).

Der sekundäre Herkunftseffekt beschreibt den Einfluss elterlicher Bildungsentscheidungen auf den kindlichen Bildungsweg. Da Familien je nach ihrem sozioökonomischen Status mit den unterschiedlichen Bildungswegen verschiedene subjektive Kosten und Nutzen verbinden, variieren die elterlichen Bildungsentscheidungen schichtspezifisch: Eltern aus höheren sozialen Schichten neigen deutlich häufiger dazu, ihr Kind auf ein Gymnasium zu schicken, als Eltern aus niedrigen Sozialschichten, da für sie die sozialen Distanzen geringer sind, die Kosten eines längeren Schulbesuchs aufgrund des höheren Einkommens weniger ins Gewicht fallen und sie überdies eher davon ausgehen können, dass ihr Kind den höheren Bildungsabschluss auch tatsächlich erreicht (Boudon 1974). Empirische Befunde zeigen jedoch, dass beispielsweise für die Übergangsentscheidung nach der Grundschule die sekundären Herkunftseffekte bei Migranten eine eher untergeordnete Bedeutung haben (Relikowski et al. 2010). So scheinen die mit einem längeren Schulbesuch verbundenen Kosten eher nachrangig zu sein. Begründen lässt sich dies mit den hohen Bildungsaspirationen eingewanderter Familien, welche dazu führen, dass unter Kontrolle der sozialen Herkunft und der schulischen Performanz für Migranten sogar höhere Übergangsraten auf das Gymnasium nachgewiesen werden können als für Kinder aus Familien ohne Migrationshintergrund (ibd.). „Für die Förderung von Migrantenkindern bedeutet dies, dass sich politische Interventionen explizit auf die Mechanismen primärer Herkunftseffekte konzentrieren müssen“ (Relikowski et al. 2010: 165). Eine besondere Bedeutung kommt hierbei einer möglichst frühen Förderung der kindlichen Fähigkeiten und Kompetenzen zu. So zeigen Kristen et al. (2010) für türkischstämmige Kinder, dass die Kompetenzentwicklung in den ersten Lebensjahren bei der Erklärung der Bildungsbenachteiligung eine Schlüsselrolle spielt. Um den Ungleichheiten im Bildungssystem entgegenzuwirken, sollte für Kinder aus Familien mit Migrationshintergrund eine Förderung „spätestens mit dem Eintritt in den Kindergarten“ (Gogolin

1 So belegen Daten des Panels Arbeitsmarkt und soziale Sicherung (PASS), dass die deutsche Sprache zwar für die Mehrheit der Familien mit Migrationshintergrund fester Bestandteil des Familienalltags ist, $80 \%$ der Familien zu Hause jedoch noch eine weitere Sprache sprechen (BMFSFJ 2010: 25f.). 
2008: 79) einsetzen. Aufgrund ihrer zentralen Rolle und ihrer besonderen Verantwortung für die kindliche Entwicklung richtet sich dieser Imperativ in besonderer Weise an die Eltern. Denn von ihnen wird die optimale Erziehung und Förderung aller kindlichen Fähigkeiten (Beck-Gernsheim 1990: 168) erwartet, um so die Basis für eine erfolgreiche Schulkarriere zu legen (Wissenschaftlicher Beirat 2002). Gleichzeitig wird jedoch eine Unterstützung der Eltern gefordert, um sie ,in ihren erzieherischen und bildungsrelevanten Kompetenzen zu stärken" (Peterander 2009: 972) sowie zu befähigen, ihren Kinder schon früh ein entwicklungsförderndes Sozialisationsfeld $\mathrm{zu}$ verschaffen (Bundesregierung 2007: 49).

\section{Familienbildung als präventives Angebot}

Die Unterstützung von Eltern bei der Erziehung und Förderung ihrer Kinder ist ein primärer Aufgabenbereich der Familienbildung. Diese ist als präventives Angebot im Kinder- und Jugendhilfegesetz (SGB VIII) bundesweit einheitlich verankert und richtet sich an alle Familien, unabhängig von ihrer Herkunft, ihrer sozialen Stellung und ihrer aktuellen Lebenssituation. Die Inanspruchnahme von Leistungen der Familienbildung ist an keinerlei Voraussetzungen gebunden.

Elternbildung ist ein Teil der Familienbildung: Zur Familienbildung als der umfassenderen Kategorie gehören alle familien- und erziehungsbezogenen Bildungsmaßnahmen, auch jene, in welche auch die Kinder einbezogen werden, während Elternbildung sich explizit an die erwachsenen Erziehungspersonen wendet (Tan 2011). Insofern sind für die Familienbildung neben dem SGB VIII auch die Erwachsenenbildungsgesetze von Bedeutung. Sie sind bundesländerspezifisch unterschiedlich ausgestaltet. Die Position der Familienbildung zwischen Kinder- und Jugendhilfe einerseits und Erwachsenenbildung andererseits berührt nicht nur Fragen der institutionellen Zuordnung und der Finanzierung familienbildender Angebote, sondern hat auch Konsequenzen für die Frage, was unter Familienbildung verstanden wird, welche Zielsetzungen familienbildende Angebote verfolgen und wie sie ihre Adressaten erreichen.

\subsection{Grundverständnis, Zielsetzungen und Handlungsmaximen von Familienbildung}

Bis heute hat sich keine allgemein akzeptierte Definition von Familienbildung und den darunter zu fassenden Angeboten durchgesetzt. Vielmehr lassen sich in der wissenschaftlichen und (bildungs)politischen Diskussion unterschiedliche Perspektiven ausmachen, die mit je eigenen Zielsetzungen, einem bestimmten Verständnis von Bildungsprozessen sowie Bildern der lernenden Personen verbunden sind (Rupp et al. 2010: 51ff.). Auf der Basis einer Sichtung existierender Begriffsdeutungen und Zielformulierungen haben Rupp et al. (2010) eine sehr breite und umfassende Definition von Familienbildung entwickelt. Danach vermittelt Familienbildung frühzeitig und lebensbegleitend Wissen über familiale Belange, unterstützt die Entwicklung bzw. den Aufbau von familienbezogenen Fähigkeiten, regt zur Reflexion an und dient der Orientierung, fördert die gesellschaft- 
liche Teilhabe und erweitert die familialen Handlungsspielräume. ${ }^{2}$ Familienbildung in diesem Sinne nimmt alle Familienmitglieder in den Blick und ist den Konzepten der Salutogenese und des Empowerment bzw. der Ressourcenorientierung verpflichtet (Rupp/ Smolka 2006). Zudem greift sie humanistische und emanzipatorische Zielsetzungen auf und zielt auf eine Handlungsbefähigung des Individuums wie der ganzen Familie im Sinne des Capability-Konzepts ${ }^{3}$ ab (Keupp 2011). Die Unterstützung von Eltern bei der frühen Förderung ihrer Kinder ist dabei als ein spezifisches Teilziel der Familienbildung zu sehen.

Als ein zentraler Schlüssel für die Akzeptanz von Familienbildung gilt Niedrigschwelligkeit. „Sollen Unterstützungsangebote möglichst viele Familien erreichen und für viele zugänglich, also niedrigschwellig sein, so müssen sie sich inhaltlich, zeitlich und örtlich an den Lebenswelten von Familien orientieren." (Deutscher Verein 2005: 6; Hervorhebung im Original) $\mathrm{Zu}$ den Kriterien für Niedrigschwelligkeit gehören u.a. Alltagsnähe, zeitliche und räumliche Erreichbarkeit, Orientierung am sozialen Raum, ein verstärkter Einsatz von Gehstrukturen, Anschlussfähigkeit sowie die Beachtung des speziellen kulturellen bzw. weltanschaulichen Hintergrundes und des Gender Mainstreaming (Deutscher Verein 2005; Rupp et al. 2010).

\subsection{Formen der Familienbildung}

Familienbildung in Deutschland zeichnet sich durch eine große Vielfalt an Formen, Inhalten und Trägern aus. Am bekanntesten ist die so genannte institutionelle Familienbildung. Sie findet in unterschiedlichen Einrichtungen statt, wie beispielsweise in Familienbildungsstätten, die ausschließlich Angebote für Familien durchführen, aber auch in Einrichtungen, die primär andere Aufgaben haben oder für die Familien eine unter mehreren Zielgruppen sind, wie z.B. Erziehungsberatungsstellen, Kindertageseinrichtungen oder Erwachsenenbildungseinrichtungen. Neben der institutionellen Familienbildung gibt es die mediale oder informative Familienbildung in Printmedien, wie z.B. Zeitschriften und Broschüren, in audiovisuellen Medien, wie z.B. Fernsehen und Radio, in Form digitaler Speichermedien wie z.B. CD oder DVD, sowie im Internet. Die informelle Familienbildung schließlich findet außerhalb institutionalisierter Zusammenhänge, beispielsweise als selbst organisierter Erfahrungsaustausch von Eltern in Familien- und Mütterzentren oder im Rahmen von Selbsthilfeinitiativen statt (Rupp et al. 2010).

\subsection{Migranten als Zielgruppe der institutionellen Familienbildung}

Prinzipiell gehören alle in Deutschland lebenden Familien und deren Mitglieder zum Adressatenkreis der Familienbildung und damit auch der institutionellen Familienbildung.

2 Eine ausführliche Version dieser Definition findet sich in Rupp et al. 2010: 61f.

3 Der „Capability Approach“ und wurde von Sen und Nussbaum entwickelt und ist ursprünglich ein wirtschaftswissenschaftlicher Ansatz. Seit einiger Zeit findet er jedoch auch Eingang in den deutschen sozial- und erziehungswissenschaftlichen Diskurs (als Überblick Robeyns 2005; Otto/Ziegler 2010). 
Obwohl die Datenlage über die Partizipation von Eltern an Angeboten der Familienbildung als „eher desolat“ (Fischer 2011: 423) bezeichnet werden kann, geht man in Fachkreisen seit Langem davon aus, dass Familien mit Migrationshintergrund in der institutionellen Familienbildung unterrepräsentiert sind (ebd., Fischer et al. 2007; Thiessen 2008). Ursachen hierfür bestehen sowohl auf Seiten der Migranten als auch auf Seiten der Einrichtungen.

Als Hemmnisse bei den Migranten werden geringe Deutschkenntnisse, negative Erfahrungen mit schulischem Lernen, Unkenntnis über das deutsche Weiterbildungssystem oder ein anderes Verständnis des elterlichen Erziehungsauftrags angeführt (Springer 2011; Fischer et al. 2007). Barrieren bei den Einrichtungen werden insbesondere in der Mittelschichtsorientierung und in der „Migrantenferne“ der institutionellen Familienbildung gesehen (Thiessen 2008): Das Problem der Mittelschichtsorientierung in der institutionellen Familienbildung ist seit langer Zeit bekannt. Sozial benachteiligte Familien werden im Vergleich zu Familien aus der gesellschaftlichen Mittelschicht durch das familienbildende Angebot deutlich weniger erreicht (Rupp et al. 2010; Mengel 2007). Der Anteil von Angehörigen sozioökonomisch schlechter gestellten Milieus an den Teilnehmenden wird mit durchschnittlich $15 \%$ beziffert (Lösel 2006). Da Familien mit Migrationshintergrund in niedrigen gesellschaftlichen Schichten überrepräsentiert sind (Geißler 2011: 243ff.; BMFSFJ 2010), gehören sie häufig zu dieser Bevölkerungsgruppe, welche von den klassischen Angeboten der Familienbildung kaum angesprochen wird. Als „Migrantenferne" beschreibt Thiessen (2008) Tendenzen einer monokulturellen Ausrichtung der institutionellen Familienbildung. So werden erst in jüngster Zeit fachliche Diskussionen um Konzepte der interkulturellen Öffnung geführt (z.B. MFKJKS 2010) und trotz ihrer Themenvielfalt spiegeln die Angebote der institutionellen Familienbildung die spezifischen Bedürfnisse von Familien mit Migrationshintergrund nur selten wider (Fischer 2011). Neben fehlenden zielgruppenspezifischen Konzepten sind es häufig auch gerade die bewährten Formen der Zielgruppenansprache, wie z.B. schriftliche Medien, mit denen sich Migranten kaum erreichen lassen (Gaitanides 2011). Vor diesem Hintergrund sowie angesichts des antizipierten Bedarfs von Familien mit Migrationshintergrund an Familienbildungsangeboten in Folge der Ergebnisse der PISA-Studien wurden in den vergangenen Jahren zahlreiche zielgruppenspezifische Angebotskonzepte entwickelt bzw. aus dem Ausland adaptiert (Springer 2011).

\section{Familienbildungskonzepte für Migranten: Ansätze und Effekte}

Familienbildende Angebote zur Unterstützung frühkindlicher Förderung, welche speziell für Migranten entwickelt wurden, lassen sich in Abhängigkeit von ihren Programmmerkmalen in unterschiedliche Kategorien einteilen.

\subsection{Kriterien zur Kategorisierung familienbildender Angebote für Migranten}

Für die Entwicklung von Kriterien zur Kategorisierung familienbildender Angebotskonzepte für Migranten gibt es nur wenige Vorarbeiten. Leseman (2008) hat frühkindliche För- 
derangebote untersucht und anhand der Kriterien „Adressaten“ und „Setting“ vier Kategorien gebildet: kindzentrierte Angebote in einer Betreuungseinrichtung, kindzentrierte Angebote, die vor allem zu Hause umgesetzt werden, eltern-/familienzentrierte sowie kindund eltern/familienzentrierte Ansätze. Aus der Perspektive der Familienbildung ist im Unterschied zu dieser Differenzierung nur eine solche zwischen eltern- bzw. eltern- und kindzentrierten Ansätzen sinnvoll, da ausschließlich kindzentrierte Programme, wie sie Leseman einbezieht, nicht zum engeren Aufgabenspektrum der Familienbildung gehören.

Das Setting eines Angebots, also der Ort, an welchem es durchgeführt wird, steht in enger Verbindung mit seiner programmatischen Struktur. Friedrich/Siegert (2009) unterscheiden in ihrer Systematik daher zwischen Komm- und Gehstruktur, wobei Angebote mit Kommstruktur in einer Einrichtung stattfinden, während Angebote mit Gehstruktur sich dadurch auszeichnen, dass die Teilnehmenden aktiv aufgesucht werden, z.B. in ihrem häuslichen Umfeld. Über die Unterscheidungskriterien „Adressaten“ und „Programmstruktur" hinaus erscheint es uns für eine aussagekräftige Kategorisierung wichtig, auch die Komplexität der Konzepte zu berücksichtigen. Denn es spielt eine bedeutsame Rolle, ob die Bildungsarbeit von Eltern bzw. von Eltern und Kindern gemeinsam im Rahmen eines Programmbausteins erfolgt, wie beispielsweise bei regelmäßig stattfindenden Eltern-Kind-Gruppentreffen, oder im Rahmen mehrerer Programmbausteine, wobei bei letzteren zu unterscheiden ist zwischen

- rein elternzentrierten Angeboten mit mehreren Bausteinen oder Modulen, wie beispielsweise regelmäßige Hausbesuche sowie regelmäßige Gruppentreffen der Eltern, und

- Angebotskonzepten, die aus getrennten Modulen für Eltern und Kinder bestehen, wie beispielsweise Gruppentreffen für Eltern und separate Förderung der Kinder.

Berücksichtigt man diese drei Unterscheidungskriterien „Komplexität“, „Adressaten“ und "Struktur" im Zusammenspiel, ergibt sich ein Kategoriensystem, welches in Abbildung 1 graphisch dargestellt ist. Insgesamt lassen sich acht Kategorien von Familienbildungskonzepten unterscheiden, wobei die Recherche entsprechender Praxisprojekte, welche im Folgenden beschrieben wird, ergab, dass nur für sechs dieser Kategorien aktuelle Projektdaten verfügbar sind. 
Abbildung 1: Analytische Kategorien für Familienbildungskonzepte

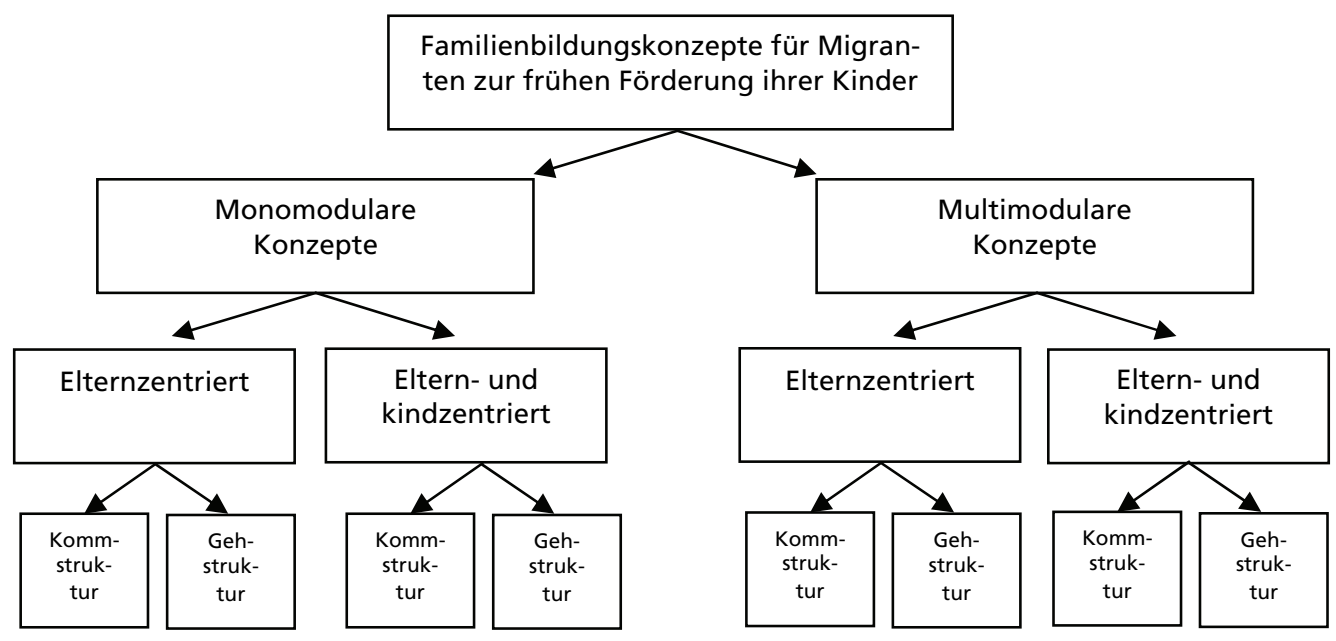

Quelle: Eigene Darstellung

\subsection{Kategorien familienbildender Konzepte für Migranten}

Zur empirischen Validierung des entwickelten Kategorienschemas wurden im Rahmen einer umfangreichen Recherche in der Fachliteratur und im Internet sowie im Zusammenhang mit eigenen (praxisbezogenen) Forschungsprojekten eine Vielzahl von Angebotskonzepten gesichtet. Für die Analyse wurden entsprechend der Fragestellung ausschließlich Angebote ausgewählt, die folgende Merkmale aufweisen:

Es handelt sich um ein Angebot

- der institutionellen Familienbildung,

- für Familien mit Migrationshintergrund ${ }^{4}$ mit Kindern bis zu sechs Jahren,

- mit mehreren Teilen und/oder Terminen sowie

- zur familienorientierten Vorbereitung der Kinder auf den formalen Bildungsweg. ${ }^{5}$

4 In den Sozialwissenschaften wird der Migrationshintergrund in der Regel anhand unterschiedlicher statistischer Merkmale, wie z.B. dem Geburtsland, der Staatsbürgerschaft und/oder der Einbürgerung, operationalisiert. In der praktischen Arbeit mit Familien orientiert man sich dagegen meist eher an weichen Indikatoren, wie z.B. an einer durch Migration geprägten Lebenswelt, an subjektiven Zuschreibungen sowie an (wahrgenommenen oder vermuteten) spezifischen Bedürfnissen. Systematische Analysen von in der Familienbildung verwendeten Definitionen von Migrationshintergrund liegen bislang nicht vor. Angesichts dieser Offenheit hinsichtlich der definitorischen Abgrenzung der Zielgruppen in der Familienbildung wird im vorliegenden Aufsatz bewusst auf eine (eigene) Definition von Migrationshintergrund verzichtet.

5 Projekte wie „FuN (Familie und Nachbarschaft)“ (Brixius et al. 2005) oder „Eltern-AG“ (Sodtke et al. 2007), deren Fokus weniger auf bildungsbezogene Aspekte, sondern eher auf die Vernetzung von Familien, die innerfamiliale Beziehungsqualität sowie die Entwicklung von Zeitstrukturen ausgerichtet ist, wurden daher nicht in die Analyse einbezogen. 
Damit konzentrieren wir uns bewusst auf einen kleinen Ausschnitt der Familienbildung, welche eigentlich - wie in Kapitel 2 dargestellt wurde - einen deutlich breiteren Anwendungsbereich hat. Trotz dieser Fokussierung stellt sich die verbleibende Angebotspalette als äußerst komplex und vielschichtig dar. So kann in Bezug auf Familienbildung generell festgestellt werden, dass viele „Angebote überwiegend relativ wenig vorstrukturiert und eher frei gestaltet werden, selbst wenn ein Kursmanual zugrunde liegt" (Lösel/Runkel 2012: 269) und wenig Informationsmaterialien verfügbar sind. Folglich erhebt die folgende Auswahl weder einen Anspruch auf Vollständigkeit noch auf Repräsentativität.

\subsubsection{Monomodulare Konzepte}

Unter monomodularen Programmkonzepten verstehen wir familienbildende Angebote, welche aus einem einzelnen Programmbaustein bestehen. Dieser kann entweder elternzentriert oder eltern- und kindzentriert konzipiert sein. Zusätzlich wird zwischen Angeboten mit Komm- und solchen mit Gehstruktur unterschieden.

\section{Elternzentrierte Konzepte mit Kommstruktur}

Programmkonzepte, die sich der Kategorie „Elternzentrierte Konzepte mit Kommstruktur" zuordnen lassen, richten sich ausschließlich an Eltern und finden in einer Einrichtung statt, welche von den Teilnehmenden aufgesucht werden muss. Dabei handelt es sich häufig um eine Einrichtung aus der Lebenswelt der Kinder, beispielsweise den Kindergarten. Im Gruppensetting werden Erziehungsthemen theoretisch und diskursiv erarbeitet und ergänzend relevante Informationen vermittelt, beispielsweise über das deutsche Bildungssystem. Teilweise sind die Erziehungsinhalte auch in einen Deutschkurs eingebunden.

Beispiele für elternzentrierte Konzepte mit Kommstruktur sind die Programme „Mama lernt Deutsch im Kindergarten“ und „Eltern-Café“. Der Kurs „Mama lernt Deutsch im Kindergarten“ wurde in Anlehnung an die in Grundschulen angebotenen Kurse „Mama lernt Deutsch - Papa auch“ des Amts für Multikulturelle Angelegenheiten der Stadt Frankfurt entwickelt (Jugendamt Nürnberg, o.J.). Durch die Kursteilnahme sollen Mütter nicht nur ihre eigenen Deutschkenntnisse verbessern, sondern auch dazu ermutigt werden, ihre Kinder bei Problemen mit der deutschen Sprache zu unterstützen und schulbezogene Entscheidungen leichter treffen zu können. ${ }^{6}$

Das Projekt „Elterncafé“ verbindet in einem sehr offenen Konzept Elternbildung mit Informationsvermittlung über soziale Einrichtungen vor Ort (Landesstiftung Baden-Württemberg 2006: 21ff.). Dabei werden die Eltern zu regelmäßigen Kaffeerunden in die Kindertagesstätte, welche ihr Kind besucht, eingeladen, um sich unter Leitung einer Fachkraft in einer offenen Diskussion über familien- und erziehungsbezogene Themen auszutauschen.

6 Im Unterschied zu den hier beschriebenen Kursen sind allgemeine Erziehungskurse für Eltern mit Migrationshintergrund thematisch deutlich breiter angelegt. Dem Thema schulische Bildung wird relativ zu den anderen Kursinhalten keine höhere Bedeutung beigemessen. Beispielhaft kann hier der Elternkurs „Starke Eltern - Starke Kinder“ für türkischsprachige Eltern des Deutschen Kinderschutzbundes sowie das Elterndiplom der RAA Gelsenkirchen angeführt werden. 
Elternzentrierte Konzepte mit Gehstruktur

Konzepte der Kategorie „Elternzentrierte Konzepte mit Gehstruktur“ richten sich ebenfalls ausschließlich an Eltern. Die Vermittlung der Bildungsinhalte erfolgt im Gegensatz zur ersten Konzeptkategorie jedoch im Haushalt der Familien. Die teilnehmenden Eltern werden über mehrere Wochen von einer Hausbesucherin begleitet. Während der Besuche erhalten sie Informationen zu Erziehungsfragen, werden ermutigt und angeleitet, den Entwicklungsprozess ihres Kindes aktiv zu unterstützen. Flankierend erhalten sie Informationen über Angebote im Sozialraum sowie über allgemeine Unterstützungsangebote. Es wird darauf geachtet, dass die Hausbesucherinnen und die von ihnen betreuten Familien einen ähnlichen kulturellen und sprachlichen Hintergrund haben, um soziale Distanzen zu reduzieren und eine muttersprachliche Information der Eltern zu ermöglichen. Für die Hausbesucherinnen selbst stellt die elternbildende Tätigkeit ebenfalls eine Qualifizierung dar.

Exemplarisch für diese Kategorie ist das Konzept „Stadtteilmütter“ des Diakonischen Werks Berlin Stadtmitte (Diakonisches Werk Berlin Stadtmitte e.V. 2009). Als „Stadtteilmütter" fungieren erwerbslose Frauen mit Migrationshintergrund, welche über gute Deutschkenntnisse verfügen. Sie werden im Rahmen einer sechsmonatigen Fortbildung auf Hausbesuche bei sozial benachteiligten Familien mit Kindern unter sechs Jahren, insbesondere bei solchen mit Migrationshintergrund, vorbereitet.

\section{Eltern- und kindzentrierte Konzepte mit Kommstruktur}

Im Gegensatz zu den ersten beiden Kategorien richten sich Konzepte der Kategorie „Eltern- und kindzentrierte Konzepte mit Kommstruktur" an Eltern und Kinder, das heißt Eltern besuchen gemeinsam mit ihren Kindern Veranstaltungen in Einrichtungen und lernen dort über mehrere Wochen in einer festen Gruppe und unter fachlicher Anleitung anwendungsbezogen spielerische Fördermöglichkeiten kennen. $\mathrm{Zu}$ unterscheiden sind dabei Konzepte zur allgemeinen Förderung der kindlichen Entwicklung und Konzepte zur kindlichen Sprachförderung, wobei insbesondere letztere hier relevant sind.

Exemplarisch für Konzepte zur kindlichen Sprachförderung sind die Programme „Sprachförderung in Eltern-Kind-Gruppen“ und „SIMPL (Sprache im Mittelpunkt)“. Das Programm „Sprachförderung in Eltern-Kind-Gruppen“ basiert auf den Konzepten „Samenspel“ und „SamenTaal“ aus den Niederlanden (Kühn 2011: 8) und richtet sich insbesondere an eher isoliert lebende neuzugewanderte Mütter mit Kindern unter drei Jahren. Im Rahmen einer Eltern-Kind-Gruppe erlernen sie, wie sie die sprachliche Förderung ihrer Kinder sowohl in Bezug auf die deutsche Sprache als auch in Bezug auf die Herkunftssprache unterstützen können. Aus diesem Grund wird die Gruppe von zwei Fachkräften geleitet, von denen eine selbst einen Migrationshintergrund hat und die Sprache der - idealerweise sprachhomogen zusammengesetzten - Gruppe spricht (Kühn 2011; Colberg-Schrader 2005).

Im Programm „SIMPL“ werden die Eltern als Beobachterinnen und auch als aktiv Handelnde in die Deutschförderung ihrer Kinder im Kindergarten einbezogen. Anwendungsbezogen erlernen sie verschiedene Möglichkeiten, wie sie Sprachaufbau und -entwicklung ihres Kindes - entweder im Deutschen oder in der Herkunftssprache - auf spielerische Weise fördern können (RAA Gelsenkirchen, o.J.). 
Tabelle 1: Exemplarische monomodulare Konzepte

\begin{tabular}{l|llll}
\hline $\begin{array}{l}\text { Adressaten } \\
\text { Struktur }\end{array}$ & Eltern & & Eltern und Kinder & \\
\hline Beispiele & Kommstruktur & Gehstruktur & Kommstruktur & Gehstruktur \\
& Mama lernt Deutsch & Stadtteilmütter & Sprachförderung in & Aktuell keine Projektda- \\
& im Kindergarten & & Eltern-Kind-Gruppen & ten verfügbar. \\
& Elterncafé & & Sprache im Mittelpunkt & \\
\hline
\end{tabular}

Quelle: Eigene Darstellung

\subsubsection{Multimodulare Programmkonzepte}

Im Gegensatz zu den monomodularen Konzepten umfassen multimodulare Programme mehrere unterschiedliche Elemente. Hierbei ist zu differenzieren zwischen elternzentrierten Konzepten (z.B. Hausbesuche und Gruppentreffen) und eltern- und kindzentrierten Konzepten sowie zwischen Konzepten mit Komm-und Gehstruktur.

\section{Elternzentrierte Konzepte mit Gehstruktur}

Multimodulare elternzentrierte Konzepte mit Gehstruktur umfassen mehrere Programmbausteine, die sich ausschließlich an die Eltern richten und von denen mindestens einer auf einer Gehstruktur basiert. Die Bildungsarbeit mit den Eltern erfolgt in unterschiedlichen Settings, so wird z.B. die individuelle Unterstützung der Eltern zu Hause durch Gruppentreffen in einer Einrichtung ergänzt. Die Bildungsarbeit wird von Fachkräften oder von geschulten Laien übernommen, wobei letztere häufig einen Migrationshintergrund haben und selbst von der aktiven Beteiligung an der Projektarbeit profitieren sollen. Ein bekanntes Beispiel für diese Angebotskategorie ist das Programmkonzept „HIPPY“ (Home Instruction for Parents of Preschool Youngsters), ein aus Israel stammendes international verbreitetes Programm für Eltern mit Kindern im Alter von vier bis sechs Jahren. Durch die Programmteilnahme lernen die Eltern Übungs- und Spielmaterialien kennen, mit denen sie ihr Kind auf den Schuleintritt vorbereiten können. Die Anleitung durch die Hausbesucherin erfolgt in Form eines Rollenspiels, in welchem z.B. die Mutter die Rolle des Kindes und die Hausbesucherin die Rolle der Mutter übernimmt (Lombard 1981). Wichtiger Bestandteil des Programms ist das tägliche Üben in der Familie zwischen den einzelnen Anleitungstreffen.

\section{Eltern- und kindzentrierte Konzepte mit Kommstruktur}

Multimodulare eltern- und kindzentrierte Programme bestehen aus gesonderten Programmbausteinen für Eltern und Kinder, die jedoch zeitlich und teilweise auch inhaltlich aufeinander abgestimmt sind. Die Förderung der Kinder in der deutschen Sprache findet im Kindergarten statt und wird von Erzieherinnen übernommen, die speziell für diese Aufgabe weiterqualifiziert wurden. Die Schulung der Eltern erfolgt in der Regel im Gruppensetting ebenfalls im Kindergarten und basiert somit auf einer Kommstruktur, wobei teilweise auch andere soziale Einrichtungen im Sozialraum einbezogen werden. Die Durchführung des Programms wird von zielgruppenangehörigen Laien mit guten Kenntnissen im Deutschen sowie in der Herkunftssprache übernommen. Diese werden in Schulungen und bei wöchentlichen Treffen durch eine Projektkoordinatorin qualifiziert und begleitet. Die Angebotsdauer und der Turnus sind je nach Konzept unterschiedlich. 
Exemplarisch für die Kategorie „Eltern- und kindzentrierte Konzepte mit Kommstruktur" sind die Programme „Rucksack Kita“ und „frühstart“. Beide ergänzen die Förderung der deutschen Sprachkompetenzen der Kinder im Kindergarten mit Elternbildung. Sie unterscheiden sich jedoch hinsichtlich der inhaltlichen Ausrichtung des elternbildnerischen Programmbausteins. Ziel des Elternbildungsmoduls von „Rucksack Kita“ ist die Unterstützung von Müttern mit Migrationshintergrund bei der Förderung und Spracherziehung ihres Kindes in der Herkunftssprache. Die Mütter erhalten in wöchentlichen Gruppentreffen ein festes Übungsprogramm, welches täglich gemeinsam mit dem Kind zu bearbeiten ist (Springer 2011; Breitkopf/Schweitzer 2005). Das Elternbildungsmodul des hessischen Programms „frühstart“ zielt hingegen darauf ab, Eltern in allgemeinen Fragen kindlicher Entwicklung und Bildung zu unterstützen, Kenntnisse über das deutsche Bildungssystem zu vermitteln sowie die Zusammenarbeit zwischen den Eltern und den Erzieherinnen des Kindergartens zu fördern (Springer 2011).

\section{Eltern- und kindzentrierte Konzepte mit Gehstruktur}

Unter die Rubrik „Multimodulare eltern- und kindzentrierte Konzepte mit Gehstruktur“ fallen Hausbesuchsprogramme, welche sich an Eltern und ihre Kinder richten, wobei letztere aktiv in die Arbeit mit den Eltern einbezogen werden. So können die Eltern beispielsweise neu kennengelernte Fördermöglichkeiten noch im Beisein der Hausbesucherin ausprobieren und vom Feedback der Hausbesucherin profitieren. Kombiniert werden die Hausbesuche in der Regel mit gemeinsamen Gruppentreffen aller am Programm teilnehmenden Eltern. Die Elternschulung wird von Fachkräften oder Laien übernommen, die im Rahmen von Schulungen und Anleitungstreffen durch eine Projektkoordinatorin für diese Aufgabe vorab und begleitend qualifiziert werden. Dabei setzen die Programme mehrheitlich auf Hausbesucherinnen der gleichen ethnischen Herkunft wie die teilnehmenden Familien, um sprachliche und kulturelle Distanzen zu reduzieren und die Akzeptanz der Hausbesucherin als Ansprechpartnerin und Vertrauensperson zu erhöhen. Die Programmkonzepte sehen in der Regel eine Laufzeit von mehreren Jahren mit einem vergleichsweise hohen Interventionsturnus vor.

Exemplarisch für diese Konzeptkategorie sind die Konzepte „PAT (Parents as Teachers)“ und „Opstapje“. Sie richten sich an Eltern mit Kindern in unterschiedlichen Altersgruppen. Das Konzept von „PAT“ wurde Anfang der 1980er Jahre in den USA für Eltern mit Kindern bis zu drei Jahren entwickelt und umfasst neben monatlichen (elternund kindzentrierten) Hausbesuchen und regelmäßigen Gruppentreffen der Eltern mindestens ein Screening pro Programmjahr zur Überprüfung der altersgerechten kindlichen Entwicklung sowie Aktivitäten zur Vernetzung der Eltern untereinander und zur Vermittlung von Informationen über andere Einrichtungen (Sindbert 2009).

Das ursprünglich in den Niederlanden entwickelte Programm „Opstapje“ (Cordus/van Oudenhoven 1997) lehnt sich an „HIPPY“ an, richtet sich jedoch an Eltern und deren Kinder im Alter von zwei bis vier Jahren und ist daher deutlich spielerischer gehalten. Die Anleitung der teilnehmenden Eltern erfolgt bei „Opstapje“ im ersten Programmjahr auf dem Konzept des Modelllernens, im zweiten Programmjahr übernimmt die Hausbesucherin die Beobachtung - und gegebenenfalls auch Korrektur - der Mutter/Vater-KindInteraktion (Sann/Thrum 2005: 29; Hable/Schneider 2003: 32ff.). 
Tabelle 2: Exemplarische multimodulare Konzepte

\begin{tabular}{l|llll}
\hline Adressaten & Eltern & & Eltern und Kinder & \\
Struktur & Kommstruktur & Gehstruktur & Kommstruktur & Gehstruktur \\
\hline Beispiele & $\begin{array}{l}\text { Aktuell keine Pro- } \\
\text { jektdaten verfügbar. }\end{array}$ & HIPPY & Rucksack Kita & PAT \\
& & frühstart & Opstapje \\
\hline
\end{tabular}

Quelle: Eigene Darstellung

\subsection{Effekte von Familienbildungsprogrammen zur frühen Förderung}

Seit einigen Jahren ist in Deutschland ein gewachsenes Bewusstsein der Relevanz empirisch gesicherter Wirksamkeitsbelege zu beobachten (Lösel et al. 2006: 141). Dennoch liegen insbesondere für den hier interessierenden Bereich der Familienbildung für Migranten zur Prävention schulischer Schwierigkeiten ihrer Kinder nur wenige empirische Studien vor. Über Evaluationsstudien einzelner Programmkonzepte hinausgehende systematische Metaevaluationen, auf deren Basis eine Ableitung allgemeinerer Befunde und Schlussfolgerungen möglich wäre, stehen noch aus. Betrachtet man die Ergebnisse vorliegender Programmevaluationen im Bereich Familienbildung für Migranten zur frühen schulbezogenen Förderung ihrer Kinder, so weisen sie insgesamt auf positive Erfahrungen bei der Umsetzung der Projektkonzepte hin, wobei zwischen der Programmakzeptanz bei der Zielgruppe einerseits und den Programmeffekten auf das elterliche Erziehungsverhalten sowie die kindliche Entwicklung andererseits zu differenzieren ist (BrooksGunn/Markman 2005).

Die Akzeptanz eines Programms ist eine triviale Grundbedingung für seine Wirksamkeit, denn nur, wenn die Zielgruppe das Angebot tatsächlich nutzt, können sich Effekte einstellen. Die vorliegenden empirischen Befunde belegen insgesamt eine recht hohe Programmakzeptanz. So zeigt beispielsweise die in den Jahren 2003 und 2004 durchgeführte Evaluation des Konzepts „Sprachförderung in Eltern-Kind-Gruppen“" knapp 100 befragten Mütter regelmäßig an den Eltern-Kind-Gruppentreffen teilgenommen haben. Auch das von 2005 bis 2007 durch das „europäische forum für migrationsstudien“ (efms) untersuchte Programm „PAT“ weist auf eine hohe Akzeptanz bei der Zielgruppe hin, wobei besonders die persönlichen Hausbesuche sehr positiv bewertet wurden (Wolf 2007). Erstaunlicherweise wird bei Programmen mit Gehstruktur, welche neben den Hausbesuchen auch Gruppentreffen für die Eltern anbieten, das Modul Gruppentreffen vergleichsweise negativ bewertet bzw. von der Zielgruppe kaum in Anspruch genommen (Bierschock et al. 2009; Wolf 2007; Sann/Thrum 2003), während bei anderen Programmen, welche ausschließlich auf einer Kommstruktur basieren, in diesem Zusammenhang kaum Akzeptanzprobleme beobachtet wurden (z.B. Breitkopf/Schweitzer 2004).

Studien, welche die Effekte familienbildender Programme auf das elterliche Erziehungsverhalten untersuchen, zeichnen ein eher uneinheitliches Bild. Die von 2005 bis 2007 vom Staatsinstitut für Familienforschung an der Universität Bamberg (ifb) durchge-

7 Die folgenden Ausführungen basieren auf einer Zusammenfassung der Ergebnisse der Evaluationsstudie, welche den Autorinnen von der Vereinigung Hamburger Kindertagesstätten gGmbH zur Verfügung gestellt wurde. Angaben zum Studiendesign waren in dieser Zusammenfassung nicht enthalten. 
führte Evaluation zur Umsetzung von HIPPY an vier bayerischen Standorten gelangte zu eher positiven Resultaten. ${ }^{8}$ Gezeigt werden konnte, dass sich durch die Programmteilnahme die Lesekultur in den Familien spürbar verbessert hatte und die Mütter von $\mathrm{Zu}$ gewinnen im Bereich der Erziehungskompetenz, der Sicherheit im Umgang mit dem Kind sowie der Kenntnisse über die Einschulung und das deutsche Schulsystem berichten (Bierschock et al. 2009). Auch die Untersuchungen von „Rucksack Kita“ und „Sprachförderung in Eltern-Kind-Gruppen" belegen, dass sich die Programmteilnahme positiv auf die Mutter-Kind-Beziehung auswirkt sowie auch die teilnehmenden Mütter selbst davon profitieren (Breitkopf/Schweitzer 2004). Die Evaluationsstudie (2001-2004) des Deutschen Jugendinstituts (DJI) sowie der Universitäten Bremen und Regensburg zu „Opstapje“ fällt hingegen weniger optimistisch aus (Sann/Thrum 2003) ${ }^{9}$ : Die Längsschnittstudie mit einem Multi-Methoden- und quasi-experimentellen Design konnte nach Programmende zwischen Programm- und Kontrollgruppe keine signifikanten Unterschiede in Bezug auf das elterliche Erziehungsverhalten feststellen (Zimmermann/Moritz 2004).

In Bezug auf die Effekte auf die kindliche Entwicklung belegt die letztgenannte Studie bei den Kindern aus der Interventionsgruppe jedoch deutliche Fortschritte im Vergleich zur Kontrollgruppe - insbesondere im kognitiven Bereich. Allerdings wurde bei einer Follow-up-Untersuchung ein Jahr später im Vergleich zur Messung am Programmende wiederum eine Verschlechterung des kognitiven und motorischen Entwicklungsstandes der Kinder festgestellt, welcher mit dem Rückfall der Eltern in alte Erziehungsmuster erklärt wurde (Lund/Erdwien 2004: 4f.). Evaluationsstudien anderer Programmkonzepte weisen ebenfalls auf positive kurzfristige Programmeffekte auf die kindliche Entwicklung hin, langfristige Effekte wurden hingegen kaum untersucht. Die Untersuchung von „Sprachförderung in Eltern-Kind-Gruppen“" kommt zu dem Ergebnis, dass die Deutschkenntnisse der Kinder, welche am Angebot teilgenommen hatten, nach Einschätzung der Erzieherinnen besser waren als die Deutschkenntnisse von nichtteilnehmenden Kindern. Besonders hervorgehoben wurde auch, dass sämtliche Kinder, die an der Eltern-Kind-Gruppe teilgenommen hatten und im Untersuchungszeitraum drei Jahre alt wurden, im Kindergarten angemeldet wurden - zumeist in der Einrichtung, in welcher auch die Gruppentreffen stattfanden. Auch die Evaluation von „Rucksack Kita“ durch die RAA (Regionale Arbeitsstelle zur Förderung von Kindern und Jugendlichen aus Zuwandererfamilien) ${ }^{10}$ aus den Jahren 1999 bis 2001 belegt positive Effekte auf die kindliche Entwicklung. Betont wurde hier, dass sich die deutschen Sprachkompetenzen der Kinder verbessert hätten, die Sprachförderung jedoch noch ausbaufähig sei (Breitkopf/Schweitzer 2004).

8 Für HIPPY liegen in Deutschland weitere Evaluationsstudien vor. Neben der Evaluation als Modellprojekt (Kiefl 1996) existieren u.a. Studien für die Umsetzung des Programms in Hamburg (BergsWinkels/Halves o.J.).

9 Eine weitere Evaluationsstudie wurde im Rahmen der Umsetzung von Opstapje in Berlin durchgeführt (Schuhmann et al., o.J.). Auf der Basis einer Elternbefragung $(\mathrm{n}=15)$ und einer Befragung der Hausbesucherinnen $(n=3)$ konnte auch diese Studie positive Programmeffekte auf die kindliche Entwicklung nachweisen. Aufgrund des anspruchsvolleren Studiendesigns wird hier jedoch ausschließlich auf die Studie des DJI in Kooperation mit den Universitäten Bremen und Regensburg Bezug genommen.

10 Die Regionalen Arbeitsstellen zur Förderung von Kindern und Jugendlichen aus Zuwandererfamilien gibt es in Nordrhein-Westfalen. Es handelt sich dabei um kommunale Einrichtungen, die durch das Land gefördert werden (www.raa.de [Stand: 2012-02-28]). 
Neben dem Mangel an Untersuchungen der langfristigen Effekte zeigt die Analyse der vorliegenden Evaluationsstudien familienbildender Angebote für Migranten, dass die Überprüfung der anvisierten Effekte nahezu ausschließlich auf der Basis subjektiver Einschätzungen von unmittelbar am Projekt Beteiligten, insbesondere den Eltern oder den Elternbegleiterinnen, erfolgt. Die überwiegend positiven Resultate sind aufgrund des zu vermutenden sozial erwünschten Antwortverhaltens jedoch nur mit Einschränkungen als Beleg einer Wirksamkeit anzusehen. ${ }^{11}$ Studien, welche den strengen Anforderungen einer Wirkungsevaluation durch ein entsprechendes methodisches Vorgehen genügen (Lengning 2010), finden sich in diesem Bereich dagegen so gut wie nicht.

Diese Befundlage in Bezug auf den Mangel an Untersuchungen der Wirksamkeit von Familienbildung mit Migranten gilt analog für das gesamte Feld der familienbildenden Angebote in Deutschland. Eine bundesweite Bestandsaufnahme präventiver, kursförmig organisierter Angebote zur Förderung der elterlichen Erziehungskompetenz für das Jahr 2004 ergab, dass nur knapp ein Prozent der durchgeführten Angebote einer Wirkungsevaluation unterzogen wurden, wobei durchgängig auf die Einbeziehung einer Kontrollgruppe verzichtet wurde (Lösel et al. 2006: 108). Eine Metaevaluation von 27 Wirksamkeitsstudien mit Kontrollgruppendesign aus den Jahren 1976 bis 2005 weist jedoch auf insgesamt positive Effekte familienbildender Maßnahmen hin. ${ }^{12}$ Bezogen auf eltern- bzw. kindbezogene Veränderungen lassen sich „moderate, aber relevante Wirkungen der untersuchten Maßnahmen“ (ibd.: 143) feststellen. ${ }^{13}$ Nachgewiesen wurde zudem ein deutlicher Zusammenhang zwischen eltern- und kindbezogenen Effekten: Programme, welche positive Effekte bei den Eltern erzielen konnten, führten auch bei den teilnehmenden Kindern zu positiveren Veränderungen. Auch einige Zeit nach der Maßnahme konnten noch Effekte nachgewiesen werden, wobei diese schwächer ausfielen als unmittelbar nach Programmabschluss (ibd.: 129ff.). Weiter zeigte die Metaanalyse, dass relativ intensive, stark übungsorientierte und durch Programmvorgaben strukturierte familienbildende Programme zu höheren Effekten führen (Lösel/Runkel 2012). Für den Bereich der Familienbildung mit Migranten bedürfen diese Zusammenhänge noch einer weiteren empirischen Überprüfung.

11 Darüber hinaus sind die Fallzahlen der Studien sehr niedrig, was insbesondere darauf zurückzuführen sein dürfte, dass in Deutschland vorrangig (kleine) Modellprojekte und selten Regelangebote evaluiert werden.

12 Ein Großteil der berücksichtigten Evaluationsstudien bezieht sich auf Erziehungskurse. Aufgrund der geringen Zahl geeigneter Studien wurden auch Evaluationen von Kursen mit einem stärker individualisierten, beratungsorientierten Fokus berücksichtigt, wobei diese streng genommen nicht mehr der primärpräventiv ausgerichteten Familienbildung zugerechnet werden können (Lösel et al. 2006).

13 Der gemessene mittlere Gesamteffekt setzt sich aus Effektstärken in verschiedenen Kriterienbereichen zusammen. Bei den elternbezogenen Maßen wurde unterschieden zwischen dem Erziehungsverhalten, dem Erleben bzgl. Erziehungssituationen, der Persönlichkeit allgemein sowie dem sozialen Umfeld der Eltern. Kindbezogene Effekte setzen sich aus sozio-emotionalen Maßen sowie kognitiven Maßen zusammen (Lösel 2006: 130). 


\section{Diskussion und Fazit}

Im Rahmen der Sichtung und Analyse von Familienbildungsprogrammen für Migranten zur Unterstützung frühkindlicher Förderung hinsichtlich ihrer Programmstruktur und ihrer anvisierten Effekte konnten unterschiedliche Kategorien identifiziert werden. Neben monomodularen Konzepten, wie z.B. Sprach- und Erziehungskursen oder Eltern-Kind-Gruppen, gibt es multimodulare und mehrstufige Programmkonzepte (Fischer 2011: 432), welche sich durch eine Verknüpfung verschiedener Systeme und Bildungsorte, beispielsweise des Kindergartens und der Familie, auszeichnen. Mehrstufige Konzepte vermitteln elternbildende Inhalte in mehreren Schritten an die Zielgruppe, z.B. „von der Multiplikatorenschulung, über die durch Laien angeleiteten Elterngruppen bis hin zur Einzelarbeit zu Hause" (Fischer 2011: 432). Bei der Recherche nach exemplarischen Konzepten fiel auf, dass für zwei der acht theoretisch herausgearbeiteten Konzeptkategorien keine aktuellen Projektdaten verfügbar waren: Monomodulare eltern- und kindzentrierte Konzepte mit Gehstruktur sowie multimodulare elternzentrierte Konzepte mit Kommstruktur finden sich in der derzeitigen Praxis offenbar nicht wieder.

Die Analyse hat zudem gezeigt, dass sich Familienbildungskonzepte für die Zielgruppe der Migranten in hohem Maß um Niedrigschwelligkeit bemühen, etwa in Bezug auf die Veranstaltungsorte, die Gestaltung der Zugänge sowie bei der Programmkonzeption und -gestaltung. So werden vorrangig alltagsnahe Orte aus den familialen Lebenswelten, wie z.B. der Kindergarten, sowohl als Veranstaltungsorte als auch zur persönlichen Zielgruppenansprache sowie einer aktiven Teilnehmerrekrutierung genutzt. Niedrigschwelligkeit zeigt sich auch in Bezug auf die Angebotsform, z.B. durch einen offenen Gestaltungsrahmen oder Hausbesuchsprogramme. Zur Zielgruppenerreichung wird zudem häufig auf die Einbindung zweisprachiger Multiplikatoren bzw. Laienhelferinnen aus der Zielgruppe gesetzt. Von einigen Konzepten wird die Herkunftssprache allerdings primär als Hilfsmittel zur leichteren Vermittlung der Inhalte eingesetzt, während andere Programme, wie z.B. „Rucksack Kita“ die Herkunftssprachen systematisch als Ressource in das Konzept einbeziehen. Durch die Anerkennung der Eltern als Experten bei der Vermittlung ihrer Herkunftssprache an das Kind wird signalisiert, dass eine Unterstützung des kindlichen Spracherwerbsprozesses auch unabhängig vom eigenen Bildungshintergrund und von eigenen Deutschkenntnissen möglich ist. Denn ,(w)enn eine Mutter nicht gut Deutsch kann, ist die Aufforderung ,Reden Sie Deutsch mit Ihrem Kind“ ein sinnloser Appell“" (Breitkopf/Schweitzer 2005). Es zeigt sich darüber hinaus, dass die Zielgruppe der Migranten in den Konzepten in der Regel nicht weiter konkretisiert wird; vielmehr werden Migranten implizit mit sozial Benachteiligten gleichgesetzt, womit einerseits die Heterogenität der Zielgruppe verkannt wird und andererseits auch kaum milieuspezifische Ressourcen und Kompetenzen wahrgenommen und unterstützt werden. „Der Wandel von der Defizitperspektive zur Ressourcenorientierung in der Familien- und Elternbildung hat die Elternbildungsprogramme für Familien mit Migrationshintergrund nur teilweise erreicht" (Tan 2011: 175). Eine stärkere Einbeziehung der kulturellen und sozialen Unterschiede als Bereicherung und als Ansatzpunkt gemeinsamer Lernprozesse steht auf konzeptioneller Ebene insofern noch aus, wäre jedoch hilfreich, um die wertschätzende und ressourcenorientierte Haltung, um die sich viele Fachkräfte in der praktischen Umsetzung bemühen, auch bereits in den Konzepten deutlich zu verankern. 
Auch wenn nur wenige methodisch anspruchsvolle Evaluationsstudien über Familienbildungsangebote zur Unterstützung von Migranten bei der frühen Förderung ihrer Kinder vorliegen, ist zudem davon auszugehen, dass eine „erwachsenengerechte Begleitung und Unterstützung für den Familienalltag“" (Rupp et al. 2010: 37) ein vielversprechender Weg ist. Darunter sind Konzepte zu verstehen, welche Eltern mit Migrationshintergrund als Subjekte in ihrer kulturellen Eingebundenheit anerkennen (Merechil 2010: 179ff.), ihre individuellen Interessen und biografischen Erfahrungen ernstnehmen und aufgreifen, sich an ihren konkreten Lebensumständen orientieren sowie an den subjektiven Lernbegründungen der Eltern anschließen. Denn Familienbildung ist in der Regel Arbeit mit Erwachsenen, die ,als solche lernen und handeln, die eigene Vorstellungen von Erziehung, eigene Lernziele und auch Lernvorlieben besitzen“ (Rupp et al. 2010: 37).

Die Frage, inwieweit Benachteiligung im formalen Bildungssystem durch Angebote der Familienbildung zur Unterstützung der Erziehungskompetenz und der frühen Förderung der Kinder abgebaut oder verhindert werden kann, kann angesichts der unzulänglichen Befundlage im deutschsprachigen Raum (noch) nicht beantwortet werden (Friedrich/Siegert 2009). Der gesellschaftspolitische Anspruch, eine Verbesserung der schulischen Chancen und der Integration von Kindern aus Familien mit Migrationshintergrund allein über die nicht-formelle (Weiter-)Bildung der Eltern zu erreichen, dürfte zudem zu unrealistischen Erwartungen an die Familienbildung führen. Überdies werden hier - wie auch in anderen Bereichen des Sozialen - Prozesse einer gesellschaftlichen Re-Familialisierung erkennbar (Oelkers/Richter 2009), durch welche die Konsequenzen sozialstruktureller Benachteiligungen zu privat bzw. innerfamilial zu bearbeitenden Problemen erklärt werden. Oelkers/Richter sehen hier die Gefahr, dass eine solche zugeschriebene Eigenverantwortung zu einer Überforderung von Familien führen könnte - insbesondere für sozial Benachteiligte.

Angebracht erscheint somit ein integriertes Maßnahmenbündel, zu welchem beispielsweise die bedarfsgerechte und zielgruppenorientierte Kombination von Familienbildungsangeboten und anderen Ansätzen vorschulischer kindzentrierter Förderung gehört. Auch sollten strukturelle Aspekte stärker in den Blick genommen werden. „Damit die frühkindliche Förderung von Kindern aus Familien mit Migrationshintergrund gelingt [...] braucht es eine kontinuierliche, intensive und multisystemische Förderung zu Hause und in den pädagogischen Einrichtungen, die [...] mit der nachfolgenden Bildungsstufe vernetzt ist." (Edelmann 2009: 17). Eine Voraussetzung hierfür ist die Initiierung und Etablierung von Kooperations- und Vernetzungsstrukturen zwischen unterschiedlichen Bildungsstufen und -einrichtungen auf kommunaler Ebene. Zur Weiterentwicklung dieses Arbeitsbereiches bedarf es darüber hinaus auch weiterer (politischer) Rahmenbedingungen, welche z.B. auch der Wissenschaft eine angemessene Überprüfung der Wirksamkeit ermöglichen.

\section{Literatur}

Beck-Gernsheim, E. (1990). Alles aus Liebe zum Kind. In: Beck, U. \& Beck-Gernsheim, E. (Hrsg.). Das ganz normale Chaos der Liebe. Frankfurt am Main: Suhrkamp, S. 135-183.

Bergs-Winkels, D. \& Halves, E. (o.J.): Kurzbericht über die Evaluation des HIPPY-Programms in Hamburg. www.hippy-deutschland.de/aktuelles/2010/Eval\%20Hippy\%20Hamburg\%20Kurzfassung.pdf [Stand: 2012-02-28]. 
Betz, T. (2006). Milieuspezifisch und interethnisch variierende Sozialisationsbedingungen und Bildungsprozesse von Kindern. In: Alt, C. (Hrsg.), Kinderleben - Integration durch Sprache? Band 4: Bedingungen des Aufwachsens von türkischen, russlanddeutschen und deutschen Kindern. Wiesbaden: VS Verlag für Sozialwissenschaften (Schriften des Deutschen Jugendinstituts: Kinderpanel).

Bierschock, K., Dürnberger, A. \& Rupp, M. (2009). Evaluation des HIPPY-Programms in Bayern. Bamberg: Staatsinstitut für Familienforschung (ifb-Materialien 3-2008).

BMFSFJ [Bundesministerium für Familie, Senioren, Frauen und Jugend] (2010). Familien mit Migrationshintergrund. Lebenssituation, Erwerbsbeteiligung und Vereinbarkeit von Familie und Beruf, www.bmfsfj.de/BMFSFJ/Service/Publikationen/publikationen,did=164794.html [Stand: 2012-03-01].

Boudon, R. (1974). Education, opportunity and social inequality. Changing prospects in Western society. New York: Wiley \& Sons.

Breitkopf, T. \& Schweitzer, H. (2005). Elternbildung und interkulturelle Sprachförderung - Stadtteilmütterprojekt. In: Tschöpe-Scheffler, S. (Hrsg.), Konzepte der Elternbildung - eine kritische Übersicht. Opladen: Verlag Barbara Budrich, S. 237-254.

Breitkopf, T. \& Schweitzer, H. (2004). Stadtteilmütter-Projekt. Interkulturelle Sprachförderung und Elternbildung im Elementarbereich. Abschlussbericht. Essen: Stadt Essen.

Brixius, B., Koerner, S. \& Piltman, B. (2005). FuN - der Name ist Programm - Familien lernen mit Spaß. In: Tschöpe-Scheffler, S. (Hrsg.). Konzepte der Elternbildung - eine kritische Übersicht. Opladen: Verlag Barbara Budrich, S. 137-159.

Brooks-Gunn, J. \& Markman, L. B. (2005). The contribution of parenting to ethnic and racial gaps in school readiness. The Future of Children, 15, 1, S. 139-168.

Bundesregierung (2007). Der nationale Integrationsplan. Neue Wege - neue Chancen. www.bundesregierung. de/Content/DE/Archiv16/Artikel/2007/07/Anlage/2007-07-12-nationaler-integration-plan.pdf; jsessionid=CBC7BBEADA1D41D7314ED8DF872EBID3.s4t2?_blob=publicationFile \&v=3 [Stand: 2012-03-01].

Colberg-Schrader, H. (2005). Sprachförderung in der Kita - Mütter und Kinder lernen zusammen. Kita aktuell 5/2005, S. 14-17.

Cordus, J. \& van Oudenhoven, N. (1997). Early intervention: Examples of practice. Averroès programmes for children - An experience to be shared. Paris: Unesco Education Sector (Monograph No. 8/1997).

Deutscher Verein [für öffentliche und private Fürsorge e.V.] (2005). Niedrigschwelliger Zugang zu familienunterstützenden Angeboten in Kommunen. www.deutscher-verein.de/05-empfehlungen/pdf/ empfehlung-niedrigschwelliger-angebote.pdf [Stand: 2012-03-08].

Diakonisches Werk Berlin Stadtmitte e.V. (2009). Stadtteilmütter in Friedrichshain-Kreuzberg. www. dw-stadtmitte.de/fileadmin/Stadtteilmuetter/PDF-Dateien/Stadtteilmuetter_Kreuzberg_10.2009.pdf [Stand: 2012-03-01].

Edelmann, D. (2009). Frühe Förderung von Kindern aus Familien mit Migrationshintergrund - der Paradigmenwechsel von Betreuung und Erziehung hin zu Bildung und Integration. www.unifr.ch/pedg/ staff/edelmann/fruehe_foerderung.pdf [Stand: 2012-05.04].

Fischer, V. (2011). Eltern- und Familienbildung. In: Fischer, V. \& Springer, M. (Hrsg.), Handbuch Migration und Familie. Schwalbach: Wochenschau Verlag, S. 419-433.

Fischer, V., Krumpholz, D. \& Schmitz, A. (2007). Zuwanderung - Eine Chance für die Familienbildung. Bestandsaufnahme und Empfehlungen zur Eltern- und Familienbildung in Nordrhein-Westfalen. Düsseldorf: Ministerium für Generationen, Familie, Frauen und Integration des Landes NordrheinWestfalen.

Friedrich, L. \& Siegert, M. (2009). Förderung des Bildungserfolgs von Migranten: Effekte familienorientierter Projekte. Nürnberg: Bundesamt für Migration und Flüchtlinge.

Gaitanides, S. (2011). Zugänge der Familienarbeit zu Migrantenfamilien. In: Fischer, V. \& Springer, M. (Hrsg.). Handbuch Migration und Familien. Schwalbach: Wochenschau Verlag, S. 323-333.

Geißler, R. (2011). Die Sozialstruktur Deutschlands. Zur gesellschaftlichen Entwicklung mit einer Bilanz zur Vereinigung. Wiesbaden: VS Verlag für Sozialwissenschaften.

Gogolin, I. (2008). Förderung von Kindern mit Migrationshintergrund im Elementarbereich. Zeitschrift für Erziehungswissenschaft, 10, Sonderheft 11/2008, S. 79-90. 
Hable, A. \& Schneider, R. (2003). Opstapje - Darstellung der Situation in Nürnberg und Bremen. In: Deutsches Jugendinstitut (Hrsg.), Eine Kultur des Aufwachsens, Potentiale und Grenzen von Opstapje. Dokumentation der Fachtagung 27./28.02.03 in Freising. www.dji.de/bibs/351_2511 tagdok. pdf [Stand: 2012-03-05].

Jugendamt Nürnberg (o.J.). Konzept des Praxisprojekts „Mama lernt Deutsch im Kindergarten“. www.jugendamt.nuernberg.de/downloads/spiki_konzept_mama.pdf [Stand: 2012.03.01].

Keupp, H. (2011). Verwirklichungschancen von Anfang an: Frühe Förderung als Beitrag zur Befähigungsgerechtigkeit. In: Robert, G., Pfeifer, K. \& Drößler, T. (Hrsg.), Aufwachsen in Dialog und sozialer Verantwortung. Wiesbaden: VS Verlag für Sozialwissenschaften, S. 49-70.

Kiefl, W. (1996). HIPPY. Bilanz eines Modellprojekts zur Integration von Aussiedler- und Ausländerfamilien in Deutschland. München: Deutsches Jugendinstitut.

Kristen, C. \& Dollmann, J. (2010). Sekundäre Effekte der ethnischen Herkunft: Kinder aus türkischen Familien am ersten Bildungsübergang. In: Becker, B. (Hrsg.), Vom Kindergarten bis zur Hochschule. Die Generierung von ethnischen und sozialen Disparitäten in der Bildungsbiographie. Wiesbaden: VS Verlag für Sozialwissenschaften, S. 117-144.

Krüger-Potratz, M. (2004). Migrantenfamilien in Forschung und Politik - zur Einführung. In: KrügerPotratz, M. (Hrsg.), Familien in der Einwanderungsgesellschaft. Göttingen: V \& R unipress, S. 7-10.

Kühn, S. (2011). Eltern mit Migrationshintergrund in die Sprachbildung einbeziehen. München: Deutsches Jugendinstitut.

Landesstiftung Baden-Württemberg (2006). Innovative Familienbildung. Modellprojekte in Baden-Württemberg. Stuttgart: Landesstiftung Baden-Württemberg.

Lengning, A. (2010). Goldstandards für einen wissenschaftlichen Nachweis der Wirksamkeit und Effektivität einer Intervention im Bereich Früher Hilfen als Voraussetzung für ihre Verbreitung. Bundesgesundheitsblatt 53/2010, S. 1056-1060.

Leseman, P. (2008). Integration braucht frühkindliche Bildung: Wie Einwandererkinder früher gefördert werden können. In: Bertelsmann Stiftung, Migrations Policy Institute (Hrsg.). Migration und Integration gestalten. Transatlantische Impulse für globale Herausforderungen. Gütersloh: Bertelsmann Stiftung, S. 125-150.

Leyendecker, B. (2008). Frühkindliche Bildung von Kindern aus zugewanderten Familien - die Bedeutung der Eltern. IMIS-Beiträge, 34/2008, S. 91-102.

Liegle, L. (2006). Bildung und Erziehung in früher Kindheit. Stuttgart: W. Kohlhammer.

Lösel, F. \& Runkel, D. (2012). Empirische Forschungsergebnisse im Bereich Elternbildung und Elterntraining. In: Stange, W., Krüger, R. Henschel, A. \& Schmitt, C. (Hrsg.), Erziehungs- und Bildungspartnerschaften. Wiesbaden: VS Verlag für Sozialwissenschaft, S. 267-278.

Lösel, F., Schmucker, M., Plankensteiner, B. \& Weiss, M. (2006). Bestandsaufnahme und Evaluation von Angeboten im Elternbildungsbereich. Abschlussbericht. Erlangen: Universität ErlangenNürnberg, Lehrstuhl Psychologie I.

Lombard, A. (1981). Success begins at home: Educational foundations for preschoolers. Lexington, MA: Lexington Books.

Lund, K. \& Erdwien, B. (2004). Opstapje Schritt für Schritt. Teilbericht 5 der wissenschaftlichen Begleitung. Entwicklungspsychologische Untersuchungen: Entwicklung der Kinder. www.opstapje.de/ docs/Teilbericht_5.pdf [Stand: 2012-03-01].

Mengel, M. (2007). Familienbildung mit benachteiligten Adressaten. Eine Betrachtung aus andragogischer Perspektive. Wiesbaden: VS Verlag für Sozialwissenschaften.

Merechil, P. (2010). Anerkennung und Befragung von Zugehörigkeitsverhältnissen. Umriss einer migrationspädagogischen Orientierung. In: Merechil, P., Castro Varela, M., Dirim, I., Kalpaka, A. \& Melter, C. (Hrsg,), Migrationspädagogik. Weinheim: Beltz, S. 179-191.

MFKJKS [Ministerium für Familie, Kinder, Jugend, Kultur und Sport des Landes Nordrhein-Westfalen] (Hrsg.) (2010). Praxisleitfaden Interkulturelle Öffnung der Familienbildung. www.familienbildungin-nrw.de/tclasen/bildungpbw/fambild/content/e2153/e2511/e2514/FBildung_Praxisleitfaden_ Interkulturelle_ffnung_NeuauflageMFKJKS2010.pdf [Stand: 2012-03-08]. 
Oelkers, P. \& Richter, M. (2009). Re-Familialisierung im Kontext post-wohlfahrtsstaatlicher Transformationsprozesse und Konsequenzen für die Soziale Arbeit. Kurswechsel 3/2009, S. 35-46.

Otto, H.-U. \& Ziegler, H. (2010). Der Capabilities-Ansatz als neue Orientierung in der Erziehungswissenschaft. In: Otto, H,-U. \& Ziegler, H. (Hrsg.), Capabilities - Handlungsbefähigung und Verwirklichungschancen in der Erziehungswissenschaft. Wiesbaden: VS Verlag für Sozialwissenschaften, S. 9-13.

Peterander, F. (2009). Bildung und Erziehung in der frühen Kindheit. Monatsschrift Kinderheilkunde 10/2009, S. 971-976.

RAA Gelsenkirchen (o.J.). SIMPL. Sprachförderung als gemeinsame Aufgabe von Kindern, Eltern, Tageseinrichtung und Grundschule. www.raa.de/fileadmin/dateien/projektlandkarte/raa-gelsenkirchen/ SIMPL.pdf [Stand: 2012-02-28].

Relikowski, I., Schneider T. \& Blossfeld, H.-P. (2010). Primäre und sekundäre Herkunftseffekte beim Übergang in das gegliederte Schulsystem: Welche Rolle spielen soziale Klasse und Bildungsstatus in Familien mit Migrationshintergrund? In: Beckers, T., Birkelbach, K., Hagenah, J. \& Rosar, U. (Hrsg.). Komparative empirische Sozialforschung. Anwendungsfelder und aktuelle Methoden in Best Practice-Studien. Wiesbaden: VS Verlag für Sozialwissenschaften, S. 143-167.

Robeyns, I. (2005). The capability approach: A theoretical survey. Journal of Human Development, 6, 1, S. 93-114.

Rupp, M., Mengel, M. \& Smolka, A. (2010). Handbuch zur Familienbildung im Rahmen der Kinderund Jugendhilfe in Bayern. Bamberg: Staatsinstitut für Familienforschung (ifb-Materialien 7-2010).

Rupp, M. \& Smolka, A. (2006). Empowerment statt Pädagogisierung - Die Bedeutung niedrigschwelliger Konzepte für die Familienbildung. In: Bauer, P. \& Brunner, E. J. (Hrsg.), Elternpädagogik. Von der Elternarbeit zur Erziehungspartnerschaft. Freiburg: Lambertus, S. 193-214.

Sann, A. \& Thrum, K. (2005). Opstapje - Schritt für Schritt. Ein präventives Spiel- und Lernprogramm für Kleinkinder aus sozial benachteiligten Familien und ihre Eltern. München: Deutsches Jugendinstitut.

Sann, A. \& Thrum, K. (2003). Wissenschaftliche Begleitung des Programms Opstapje - mehr als trockene Zahlen. In: Deutsches Jugendinstitut (Hrsg.), Eine Kultur des Aufwachsens, Potentiale und Grenzen von Opstapje. Dokumentation der Fachtagung 27./28.02.03 in Freising. www.dji.de/ bibs/351_2511tagdok.pdf [Stand: 2012-03-05].

Sindbert, R. (2009). PAT - Mit Eltern lernen. Bessere Bildungschance für Migrantenkinder durch frühe Förderung und Elternempowerment. Migration und Soziale Arbeit 2/2009, S. 88-91.

Schuhmann, M. \& Willenbrink, M. (o.J.). Opstapje - Schritt für Schritt. Zusammenfassung der Ergebnisse der wissenschaftlichen Begleitung und Evaluation des Opstapjeprojektes in Berlin-Lichtenberg. www.opstapje.de/aktuelles/2010/Evaluation_Opstapje_Freigabe_Autor.pdf [Stand: 2012-0222].

Sodtke, D. \& Armbruster, M. (2007). Eltern-AG - Die niedrigschwellige Elternschule für die frühe Kindheit. Praxis der Kinderpsychologie und Kinderpsychiatrie 56 (2007), 8, S. 707-720.

Springer, M. (2011). Elterntrainings und Familienbildung. In: Fischer, V. \& Springer, M. (Hrsg.), Handbuch Migration und Familien. Schwalbach: Wochenschau Verlag, S. 473-501.

Tan, D. (2011). Elternbildung. In: Marschek, B. \& Brinkmann, H. U. (Hrsg.). Handbuch Migrationsarbeit. Wiesbaden: VS Verlag für Sozialwissenschaften, S. 169-178.

Thiessen, B. (2008). Wie erreicht Familienbildung und -beratung muslimische Familien? Eine Handreichung. Berlin: Bundesministerium für Familie, Senioren, Frauen und Jugend.

Ulich, M., Oberhuemer, P. \& Soltendieck, M. (2001). Die Welt trifft sich im Kindergarten. Interkulturelle Arbeit und Sprachförderung. Weinheim: Beltz.

Walter, O. (2008). Herkunftsassoziierte Disparitäten im Lesen, der Mathematik und den Naturwissenschaften: ein Vergleich zwischen PISA 2000, PISA 2003 und PISA 2006. In: Zeitschrift für Erziehungswissenschaft 10/2008, S. 149-168.

Wissenschaftlicher Beirat für Familienfragen (2002). Die bildungspolitische Bedeutung der Familie Folgerungen aus der PISA-Studie. Berlin: Bundesministerium für Familie, Senioren, Frauen und Jugend. 
Wolf, R. (2007). Spielend lernen in Familie und Stadtteil. Endbericht zur Evaluation des Programms. Bamberg: europäisches forum für migrationsstudien.

Zimmermann, P. \& Moritz, M. (2004). Ergebnisse der wissenschaftlichen Evaluation des Interventionsprojekts Opstapje. Mütterliche Anleitungsqualität und Verhalten des Kindes in einer Spielsituation. www.intern.dji.de/bibs/321_4173Vortrag_Zimmermann_Moritz_Abschlusstagung.pdf [Stand: 201202-27].

Eingereicht am/Submitted on: 12.02.2012

Angenommen am/Accepted on:06.07.2012

Anschrift der Autorinnen/Address of the authors:

Lena Friedrich, Diplom-Soziologin (korrespondierende Autorin/corresponding author)

Dr. Adelheid Smolka

Staatsinstitut für Familienforschung

an der Universität Bamberg (ifb)

Heinrichsdamm 4

96047 Bamberg

Deutschland/Germany

E-Mail: lena.friedrich@ifb.uni-bamberg.de adelheid.smolka@ifb.uni-bamberg.de 